\title{
Polarization properties of longitudinally inhomogeneous dichroic medium
}

\author{
I.S. Kolomiets, S.N. Savenkov, Ye.A. Oberemok \\ Taras Shevchenko Kyiv National University, 4, Glushkov Ave, 03127 Kyiv, Ukraine \\ Phone: +38(044)-526-0580, e-mail: kolomiets55@gmail.com
}

\begin{abstract}
Polarization properties of longitudinal inhomogeneous medium with linear and elliptical dichroism based on differential Jones matrix are considered. Properties of privileged polarizations have been analyzed. The integral Jones and Mueller matrices of media are obtained as a solution of the vector transfer equation. Orthogonalization properties of these classes of media are studied. Evolution of totally polarized light in longitudinal inhomogeneous medium with linear and elliptical dichroism has been analyzed.
\end{abstract}

Keywords: Jones matrix, Mueller matrix, dichroism, privileged polarization states, orthogonalization properties, evolution of polarization state.

Manuscript received 02.12.14; revised version received 11.03.15; accepted for publication 27.05.15; published online 08.06.15.

\section{Introduction}

Liquid crystals, which twenty years ago were quite exotic object of research [1-3], today have many important practical applications. In particular, the cholesteric and twisted nematic types of liquid crystals are widely used to create a variety of displays [4-6]. From the polarimetric viewpoint, these crystals are the class of nondepolarizing, longitudinally inhomogeneous media [7].

Jones and Mueller matrix methods $[8,9]$ to describe the polarization properties of such class of media were first used in the works $[10,11]$. Description of media based on their equivalent representation by sequence of layers each of which is characterized by corresponding differential Jones or Mueller matrices [12]. Further analysis of properties inherent to these media is based on the study of evolution of polarized light $[13,14]$. To date, we see growth of interest in studying of these media. In particular, recently a number of papers considering the different polarization properties of longitudinally inhomogeneous media with linear and elliptical birefringence have been published [15-17]. The case when the molecular plane in this medium is simultaneously characterized by linear birefringence and linear dichroism was also considered [18].

The main goal of this paper is to consider propagation of polarized light in longitudinally inhomogeneous medium characterized by linear and elliptical dichroism.

\section{The equivalent representation of the medium}

The equivalent representation of longitudinally inhomogeneous medium with amplitude anisotropy is similar to the case of longitudinally inhomogeneous medium with phase anisotropy $[13,17]$. However, in this case one monomolecular plane is thin (the thickness is 
much smaller than the longitudinal dimension of the medium) linear or elliptical partial polarizer. If each of these polarizers is linear, then they are characterized by the parameter $\xi_{0}=\frac{2 \pi}{\lambda}\left(k_{o}-k_{e}\right)$. If the polarizers are elliptical, then they are also characterized by the parameter $r_{0}=\frac{2 \pi}{\lambda}\left(k_{l}-k_{r}\right)$. These parameters are specific relative absorption between linear and circular eigenpolarizations per unit length, $k_{o}, k_{e}, k_{r}, k_{l}$ absorption coefficients of ordinary, extraordinary, right and left circular eigenpolarizations respectively. In the direction of light propagation along the axis perpendicular to the input surfaces of polarizers (molecular planes) each subsequent polarizer is rotated relatively to the previous one at the equal angle $\theta_{0}$ :

$\theta_{0}=\frac{2 \pi}{p}$

where $p$ is a step of helical structure of the medium, i.e., the smallest distance between the planes with the same orientation of molecules. Then, molecular plane orientation for the thickness $z$ can be determined as:

$\theta=\theta_{0} z$

The axis $z$ coincides with the axis of twist in the layered medium (helical axis). It should be noted that, by analogy to the case of longitudinally inhomogeneous medium with birefringence, the equation (2) is valid for longitudinally inhomogeneous medium with dichroism under the following conditions: $\left|\tan \left(2 \xi_{0}\right) / \sin \left(\theta_{0}\right)\right|<<1$ - for linear dichroism and $\left|\tan \left(2 \sqrt{\xi_{0}^{2}+r_{0}^{2}}\right) / \sin \left(\theta_{0}\right)\right|<<1$ for elliptic dichroism.

\section{Matrix models of medium under consideration}

Anisotropic properties of one molecular layer in this medium are described by the differential Jones matrix. This matrix can be obtained in a similar way to that presented in [13] for the case of longitudinally inhomogeneous medium with birefringence. In circular basis [19], the differential Jones matrices of longitudinally inhomogeneous medium with linear $(L D)$ and elliptical $(E D)$ dichroism are as follows:

$$
\begin{aligned}
& N^{L D}=\left[\begin{array}{cc}
0 & i \frac{1}{2} \xi_{0} e^{2 i \theta_{0} z} \\
i \frac{1}{2} \xi_{0} e^{-2 i \theta_{0} z} & 0
\end{array}\right], \\
& N^{E D}=\frac{1}{2}\left[\begin{array}{cc}
-r_{0} & \xi_{0} e^{2 i \theta_{0} z} \\
\xi_{0} e^{-2 i \theta_{0} z} & r_{0}
\end{array}\right] .
\end{aligned}
$$

For the transition from circular to linear bases related with the Cartesian rectangular coordinate system, one can use the following relation [19]:
$N_{\text {Lin }}=F N_{C i r} F^{-1}$,

where $F$ - transition matrix of the form:

$F=\left[\begin{array}{cc}1 & 1 \\ -i & i\end{array}\right]$

As a result, using Eqs. (3)-(6), one can obtain the following differential Jones matrices for classes of media under consideration in the linear basis:

$N_{\text {Lin }}^{L D}=\left[\begin{array}{cc}\frac{1}{2} \xi_{0} \cos \left(2 \theta_{0} z\right) & \frac{1}{2} \xi_{0} \sin \left(2 \theta_{0} z\right) \\ \frac{1}{2} \xi_{0} \sin \left(2 \theta_{0} z\right) & -\frac{1}{2} \xi_{0} \cos \left(2 \theta_{0} z\right)\end{array}\right]$

$N_{\text {Lin }}^{E D}=\left[\begin{array}{cc}\frac{1}{2} \xi_{0} \cos \left(2 \theta_{0} z\right) & \frac{1}{2}\left(-i r_{0}+\xi_{0} \sin \left(2 \theta_{0} z\right)\right) \\ \frac{1}{2}\left(i r_{0}+\xi_{0} \sin \left(2 \theta_{0} z\right)\right) & -\frac{1}{2} \xi_{0} \cos \left(2 \theta_{0} z\right)\end{array}\right]$.

Using Ref. [11], the differential Mueller matrices corresponding to the Jones matrices described by Eqs. (7) and (8) can be calculated:

$\begin{aligned} m_{\text {Lin }}^{L D} & =\left[\begin{array}{cccc}0 & \xi_{0} \cos \left(2 \theta_{0} z\right) & \xi_{0} \sin \left(2 \theta_{0} z\right) & 0 \\ \xi_{0} \cos \left(2 \theta_{0} z\right) & 0 & 0 & 0 \\ \xi_{0} \sin \left(2 \theta_{0} z\right) & 0 & 0 & 0 \\ 0 & 0 & 0 & 0\end{array}\right], \quad(9) \\ m_{\text {Lin }}^{\text {ED }} & =\left[\begin{array}{cccc}0 & \xi_{0} \cos \left(2 \theta_{0} z\right) & \xi_{0} \sin \left(2 \theta_{0} z\right) & r_{0} \\ \xi_{0} \cos \left(2 \theta_{0} z\right) & 0 & 0 & 0 \\ \xi_{0} \sin \left(2 \theta_{0} z\right) & 0 & 0 & 0 \\ r_{0} & 0 & 0 & 0\end{array}\right],\end{aligned}$

The integral polarization properties of these classes of media with the microscopic thickness $z$ can be described by the integral Jones matrix $T$. To calculate this matrix, we use the vector transfer equation [11]:

$\frac{d E}{d z}=N E$,

where $E$ is $2 \times 1$ Jones vector describing the polarization of light.

Substituting the differential Jones matrices (7), (8) in Eq. (11) and solving them with initial conditions $E_{1}(0)=E_{01} ; E_{2}(0)=E_{02}$, the elements of integral Jones matrices can be found using the equations [20]:

$T_{11}=\left.\frac{E_{1}}{E_{01}}\right|_{E_{02}=0}, \quad T_{12}=\left.\frac{E_{1}}{E_{02}}\right|_{E_{01}=0}$,
$T_{21}=\left.\frac{E_{2}}{E_{01}}\right|_{E_{02}=0}, \quad T_{22}=\left.\frac{E_{2}}{E_{02}}\right|_{E_{01}=0}$.

Then, for classes of media under consideration the integral Jones matrices are as follows: 


$$
\begin{aligned}
& T^{L D}= \\
& =\frac{1}{A}\left[\begin{array}{l}
A C\left(\theta_{0} z\right) C_{1}\left(\frac{z A}{2}\right)+\left(\xi_{0} C\left(\theta_{0} z\right)+2 \theta_{0} S\left(\theta_{0} z\right)\right) S_{1}\left(\frac{z A}{2}\right) \\
A C_{1}\left(\frac{z A}{2}\right) S\left(\theta_{0} z\right)+\left(\xi_{0} S\left(\theta_{0} z\right)+2 \theta_{0} C\left(\theta_{0} z\right)\right) S_{1}\left(\frac{z A}{2}\right)
\end{array}\right. \\
& \left.-A C_{1}\left(\frac{z A}{2}\right) S\left(\theta_{0} z\right)+\left(\xi_{0} S\left(\theta_{0} z\right)+2 \theta_{0} C\left(\theta_{0} z\right)\right) S_{1}\left(\frac{z A}{2}\right)\right], \\
& \left.A C\left(\theta_{0} z\right) C_{1}\left(\frac{z A}{2}\right)+\left(-\xi_{0} C\left(\theta_{0} z\right)+2 \theta_{0} S\left(\theta_{0} z\right)\right) S_{1}\left(\frac{z A}{2}\right)\right]
\end{aligned}
$$

$T^{E D}=$

$=\frac{1}{B}\left[\begin{array}{l}B C\left(\theta_{0} z\right) C_{1}\left(\frac{z B}{2}\right)+\left(\xi_{0} C\left(\theta_{0} z\right)+\left(-i r_{0}+2 \theta_{0}\right) S\left(\theta_{0} z\right)\right) S_{1}\left(\frac{z B}{2}\right) \\ B C_{1}\left(\frac{z B}{2}\right) S\left(\theta_{0} z\right)+\left(i\left(r_{0}+2 i \theta_{0}\right) C\left(\theta_{0} z\right)+\xi_{0} S\left(\theta_{0} z\right)\right) S_{1}\left(\frac{z B}{2}\right)\end{array}\right.$

$\left.-A C_{1}\left(\frac{z B}{2}\right) S\left(\theta_{0} z\right)+\left(\left(-i r_{0}+2 \theta_{0}\right) C\left(\theta_{0} z\right)+\xi_{0} S\left(\theta_{0} z\right)\right) S_{1}\left(\frac{z B}{2}\right)\right]$

$B C\left(\theta_{0} z\right) C_{1}\left(\frac{z B}{2}\right)+\left(\xi_{0} C\left(\theta_{0} z\right)+i\left(r_{0}+2 \theta_{0}\right) S\left(\theta_{0} z\right)\right) S_{1}\left(\frac{z B}{2}\right)$

where $\quad A=\sqrt{\xi_{0}^{2}-4 \theta_{0}^{2}}, \quad B=\sqrt{\xi_{0}^{2}+\left(r_{0}+2 i \theta_{0}\right)^{2}}$, $C(x)=\cos (x), S(x)=\sin (x), C_{1}(x)=\operatorname{ch}(x), S_{1}(x)=\operatorname{sh}(x)$.

Similarly, using the Stokes vector transfer equation for differential Mueller matrices, see [11, 19], the corresponding integral Mueller matrices can determined.

\section{The features of privileged polarizations}

In this section, to describe polarization we use the concept of a polarization complex variable $\chi$ defined as the ratio of the Jones vector components $\chi=E_{y} / E_{x}$. To describe evolution of polarization, we use the differential transfer equation in the complex variable terms [19]:

$d \chi / d z=-N_{12} \chi^{2}+\left(N_{22}-N_{11}\right) \chi+N_{21}$.

Substituting in Eq. (15) the elements of differential Jones matrices (in circular basis), Eqs. (3) and (4), one can obtain the corresponding equations of polarization evolution for longitudinally inhomogeneous medium with linear and elliptical dichroism:

$$
\begin{aligned}
& d \chi / d z=-\left(\frac{1}{2} \xi_{0} e^{2 i \theta_{0} z}\right) \chi^{2}+\frac{1}{2} \xi_{0} e^{-2 i \theta_{0} z}, \\
& d \chi / d z=r_{0} \chi-\frac{1}{2} \xi_{0} e^{-2 i \theta_{0} z}\left(-1+e^{4 i \theta_{0} z} \chi^{2}\right) .
\end{aligned}
$$

The equations (16) and (17) have the following partial solutions:

$$
\begin{aligned}
& \chi_{1,2}=K_{1,2} e^{-2 i \theta_{0} z}, \\
& \chi_{3,4}=K_{3,4} e^{-2 i \theta_{0} z},
\end{aligned}
$$

respectively. Eqs. (18) and (19) describe evolution of polarizations characterized by complex variables $K_{1,2}$ and $K_{3,4}$ with the coordinate $z$ propagating in longitudinally inhomogeneous media with linear and elliptical dichroism, respectively. The azimuths of these polarizations are changed linearly with the coordinate $z$ (there is gradual rotation from one to another molecular layer). Whereas, the ellipticity remains unchanged, when light propagates in the medium. So, according to the classification presented in [13], [14] and [19] the polarizations described by complex variables $K_{1,2}$ and $K_{3,4}$ call privileged polarizations. They propagate in longitudinally inhomogeneous medium characterized by linear $\left(K_{1,2}\right)$ or elliptical $\left(K_{3,4}\right)$ dichroism as in optically active medium with the values of optical activity $\varphi=\theta_{1,2}$ $\left(\theta_{1,2}\right.$ - azimuth of polarizations states variables $K_{1,2}$ and $K_{3,4}$ ). These complex variables are the solutions of the characteristic equations (16) and (17):

$$
\begin{aligned}
& K_{1,2}=\frac{2 i \theta_{0}}{\xi_{0}} \pm \sqrt{\left(\frac{2 i \theta_{0}}{\xi_{0}}\right)^{2}+1}, \\
& K_{3,4}=\frac{r_{0}+2 i \theta_{0}}{\xi_{0}} \pm \sqrt{\left(\frac{r_{0}+2 i \theta_{0}}{\xi_{0}}\right)^{2}+1}
\end{aligned}
$$

From the equations (20) and (21), it follows that if $r_{0}=0$, then $K_{1,2}=K_{3,4}$.

To calculate the azimuths and ellipticity of polarizations in circular basis, the following equations can be used [19]:

$\theta_{1,2}=\frac{1}{2} \arg (K)$,

$\varepsilon_{1,2}=\arctan \left(\frac{|K|-1}{|K|+1}\right)$.

As a result, azimuths and ellipticity for polarizations $K_{1,2}$ are as follows:

$$
\begin{aligned}
& \theta_{1,2}=\frac{1}{2} \arg \left(\frac{2 i \theta_{0} \pm \sqrt{\xi_{0}^{2}-4 \theta_{0}^{2}}}{\xi_{0}}\right), \\
& \varepsilon_{1,2}=\arctan \left(\frac{\left|\sqrt{1-\left(2 \theta_{0} / \xi_{0}\right)^{2}} \pm\left(2 i \theta_{0} / \xi_{0}\right)\right|-1}{\left|\sqrt{1-\left(2 \theta_{0} / \xi_{0}\right)^{2}} \pm\left(2 i \theta_{0} / \xi_{0}\right)\right|+1}\right) .
\end{aligned}
$$

Analyzing the expressions (24), (25) and using the conditions of polarization's orthogonality $K_{1,2}{ }^{*}=-1$, we should consider three cases:

1) if $4 \theta_{0}^{2}<\xi_{0}^{2}$, then $K_{1,2}$ are two non-orthogonal linear polarizations;

2) if $4 \theta_{0}^{2}=\xi_{0}^{2}$, then $K_{1}=K_{2}$ are two coincident linear polarizations with orientation $\theta_{1}=\theta_{2}=45^{\circ}$.

$3)$ if $4 \theta_{0}^{2}>\xi_{0}^{2}$, then $K_{1,2}$ are two non-orthogonal elliptical polarizations with same orientation $\theta_{1}=\theta_{2}=45^{\circ}$.

In the case of elliptical dichroism, the azimuths and ellipticity for polarizations $K_{3,4}$ differ from polarizations 
$K_{1,2}$ : in Eqs. (24) and (25), we have the sum $r_{0}+2 i \theta_{0}$ instead of the parameter $2 i \theta_{0}$. As a result, the class of media with elliptical dichroism is always characterized by elliptical non-orthogonal privileged polarizations. It should also be noted that, for both considered classes of media, the privileged polarizations are orthogonal only if $\theta_{0}=0$. (i.e. when the medium is homogeneous). In this case, the privileged polarizations are eigenpolarizations of corresponding homogeneous medium.

Comparing the features of the privileged polarizations for longitudinally inhomogeneous media with linear and elliptical dichroism with relevant properties of longitudinally inhomogeneous media with linear and elliptical birefringence [13, 17], some differences can be noted. In particular, unlike the case of birefringence for the case of dichroism the privileged polarizations are always non-orthogonal and its azimuths do not coincide with the azimuth of dichroism in the input molecular plane.

\section{Orthogonalization properties}

Orthogonalization properties of the medium are the ability of the medium to transform some input polarization to orthogonal one [21]. As it was demonstrated in $[21,22]$, for existence of orthogonalization properties in the medium, the following two conditions have to be fulfilled:

$$
\left\{\begin{array}{l}
F \geq 0 \\
R_{0}^{2} \geq 0
\end{array}\right.
$$

where $F$ and $R_{0}^{2}$ - parameters which are the functions of integral Jones matrix elements $T_{i j}$. The dependences of $F$ and $R_{0}^{2}$ on $T_{i j}$ are presented in $[15,17,22]$. Substituting the elements of Jones matrix Eq. (13) in these dependences, we get dependences of the value $F$ on (a) the parameters of anisotropy $\xi_{0}, \theta_{0}$ and (b) parameter $\theta_{0}$ at $\xi_{0}=1.8(1 / \mathrm{mm})$ for longitudinally inhomogeneous medium with linear dichroism. Results are presented in Fig. 1.

As can be seen from Fig. 1, for given the values of anisotropy parameters $\xi_{0}=1.8(1 / \mathrm{mm}), \quad \theta_{0}=$ $0.59(\mathrm{rad} / \mathrm{mm})$, this class of media will have orthogonalization properties for the thickness $z=2 \mathrm{~mm}$. In this case, $R_{0}^{2}=0.41>0$.

With account of the above values of anisotropy, to determine the polarizations that are orthogonalized by this class of media, we will analyze the product $\chi_{\text {inp }} \chi_{\text {out }}^{*}=f\left(\theta_{\text {inp }}, e_{\text {inp }}\right)\left(\chi_{\text {inp }}, \chi_{\text {out }}-\right.$ complex variables describing input and output polarizations) defined basing on the approach presented in [15] and [17]. The results are shown in Fig. 2.

It can be seen from Fig. 2 that in longitudinally inhomogeneous medium with linear dichroism two linear polarizations with azimuths $\theta_{i n p 1}=-46.12^{\circ}, \theta_{\text {inp } 2}=$ $-66.75^{\circ}$ are orthogonalized.
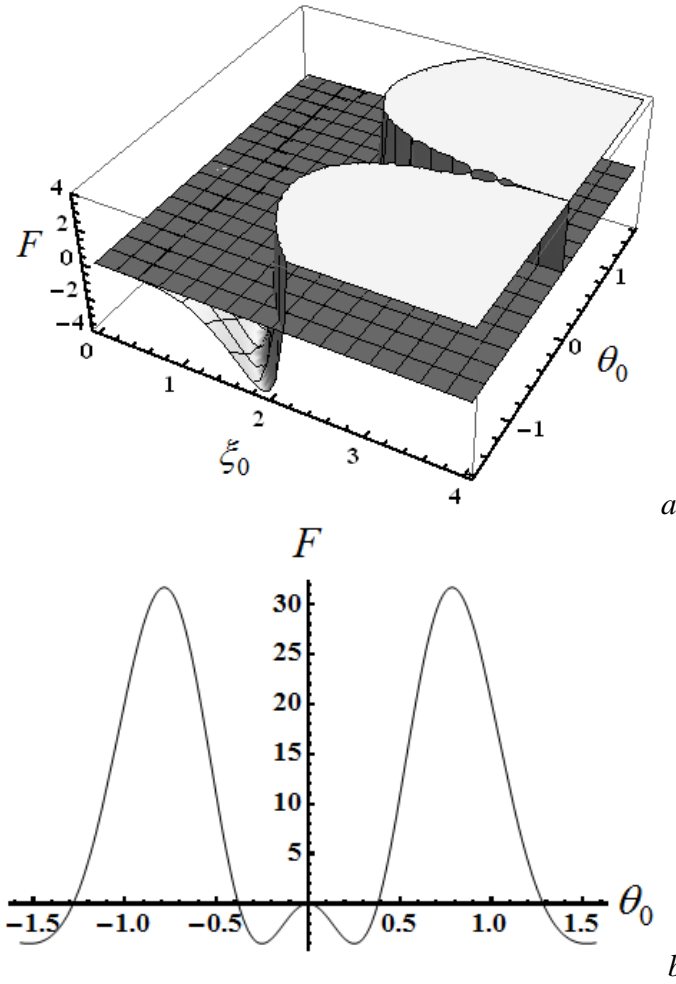

Fig. 1. Dependence of the function $F$ on the anisotropy parameters: (a) $\xi_{0}, \theta_{0}$; (b) $\theta_{0}, \xi_{0}=1.8(1 / \mathrm{mm})$ for medium with inhomogeneous linear amplitude anisotropy.

The similar analysis is carried out for orthogonalization properties of longitudinally inhomogeneous media with elliptical dichroism. In particular, taking the values of anisotropy parameters $\xi_{0}=1.8(1 / \mathrm{mm}), r_{0}=0.7(1 / \mathrm{mm}), \theta_{0}=-0.6(\mathrm{rad} / \mathrm{mm})$, and thickness $z=2 \mathrm{~mm}$, this class of media orthogonalizes the elliptical polarizations with parameters $\theta_{i n p 1}=-55.4^{\circ}$ and $e_{i n p}=-16^{\circ}$.

\section{Evolution of linear polarized light}

In the previous two sections, we examined the properties of two special types of polarizations: privileged and those orthogonalized in longitudinally inhomogeneous medium with amplitude anisotropy. Now consider what happens with other types of input polarizations, when they propagate along the helical axis in this class of media.

Let us consider this problem for linear polarization with the azimuth $\theta_{i n p}$ described by the Stokes vector [19]:

$S_{i n p}=\left(\begin{array}{llll}1 & \cos 2 \theta_{i n p} & \sin 2 \theta_{i n p} & 0\end{array}\right)^{T}$.

For that, we use the Stokes vector transfer equation of the following form [11]:

$\frac{d S}{d z}=m S$. 

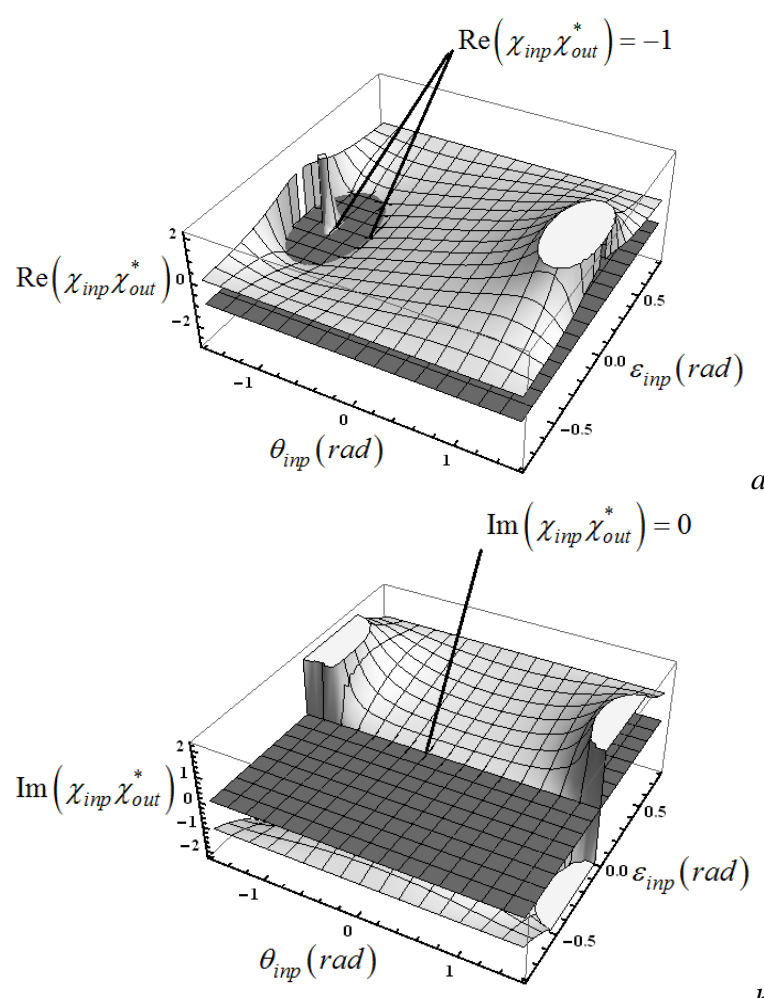

$b$

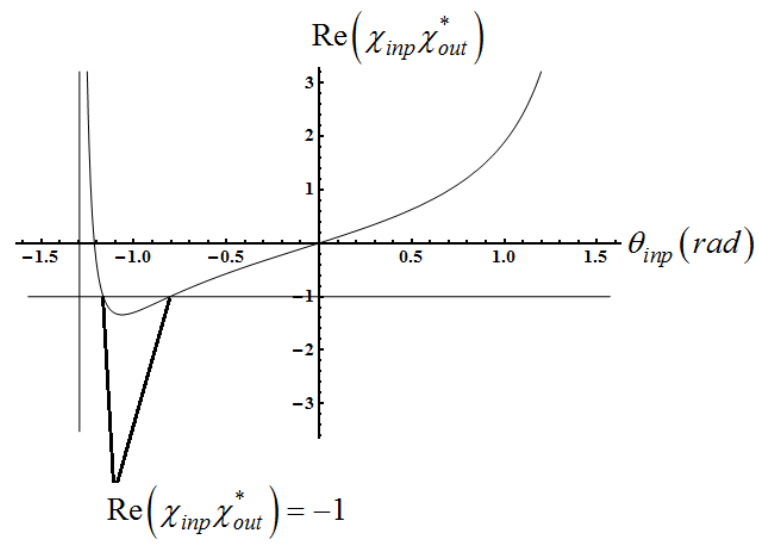

Fig. 2. Dependences of the product $\chi_{\text {inp }} \chi^{*}$ out on the azimuth $\theta_{\text {inp }}$ and ellipticity $\varepsilon_{i n p}$ of input light for medium with parameters $\xi_{0}=1.8(1 / \mathrm{mm}), \theta_{0}=0.59(\mathrm{rad} / \mathrm{mm}), z=2 \mathrm{~mm}$ : (a) dependence of $\operatorname{Re}\left(\chi_{\text {inp }} \chi_{\text {out }}\right.$ ) on $\theta_{\text {inp }}$ and $\varepsilon_{\text {inp }}$; (b) dependence of $\operatorname{Im}\left(\chi_{\text {inp }} \chi^{*}{ }_{\text {out }}\right)$ on $\theta_{\text {inp }}$ and $\varepsilon_{\text {inp }}$, (c) dependence of $\operatorname{Re}\left(\chi_{\text {inp }} \chi^{*}{ }_{\text {out }}\right)$ on $\theta_{i n p}$ at $\varepsilon_{i n p}=0$.

Substituting the differential Mueller matrices, Eqs. (9) and (10), describing longitudinally inhomogeneous medium with linear and elliptical dichroism in Eq. (28), and solving it with initial conditions Eq. (27), we obtain:

$S_{\text {out }}(z)=f\left(\xi_{0}, \theta_{0}, \theta_{\text {inp }}, z\right)$,

$S_{\text {out }}(z)=f\left(\xi_{0}, \theta_{0}, r_{0}, \theta_{\text {inp }}, z\right)$,

where $S_{\text {out }}(z)$ is the output Stokes vector.
To analyze evolution of polarization and the intensity of light with linear polarization (27), we use the equations:

$$
\begin{aligned}
& \theta_{\text {out }}(z)=\frac{1}{2} \operatorname{arctg}\left(\frac{S_{\text {out }(3)}}{S_{\text {out }(2)}}\right), \\
& \varepsilon_{\text {out }}(z)=\frac{1}{2} \operatorname{arctg}\left(\frac{S_{\text {out }(4)}}{\sqrt{S_{\text {out }(2)}^{2}+S_{\text {out }(3)}^{2}}}\right), \\
& I_{\text {out }}(z)=S_{\text {out }(1)}, \quad(32)
\end{aligned}
$$

where $\theta_{\text {out }}, \varepsilon_{\text {out }}$ and $I_{\text {out }}$ are the azimuth, ellipticity and intensity (normalized by input intensity $S_{\text {inp (1) }}$ ) of light in the output of medium with the thickness $z$. The results are presented in Figs. 3 and 4.
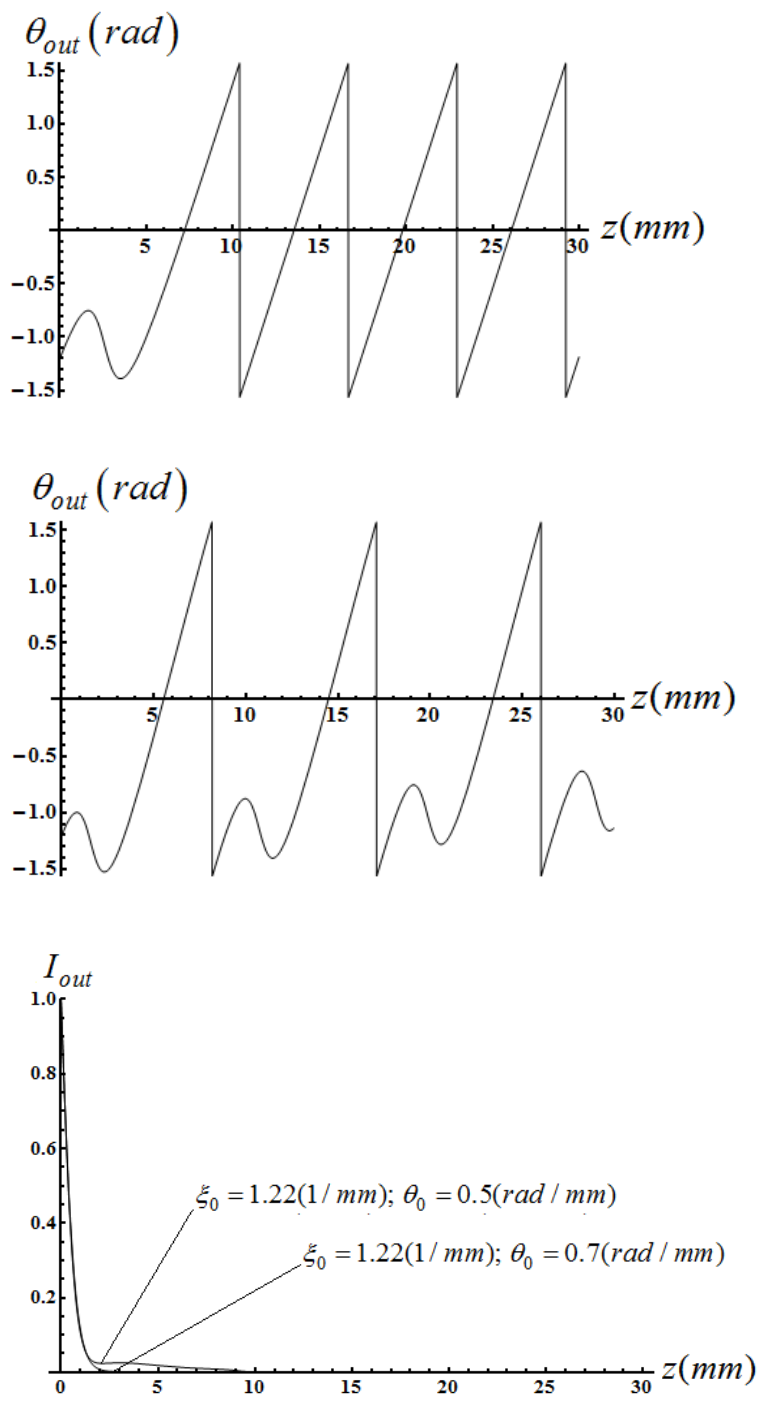

Fig. 3. Evolution of linear polarization with azimuth $\theta_{\text {inp }}=$ $-1.2(\mathrm{rad} / \mathrm{mm})$ in longitudinally inhomogeneous medium with linear amplitude anisotropy: (a) evolution of the azimuth for the case $\xi_{0}=1.22(1 / \mathrm{mm}), \theta_{0}=0.5(\mathrm{rad} / \mathrm{mm})$; (b) evolution of the azimuth for the case $\xi_{0}=1.22(1 / \mathrm{mm}), \theta_{0}=0.7(\mathrm{rad} / \mathrm{mm})$, and (c) evolution of the intensity for both cases. 

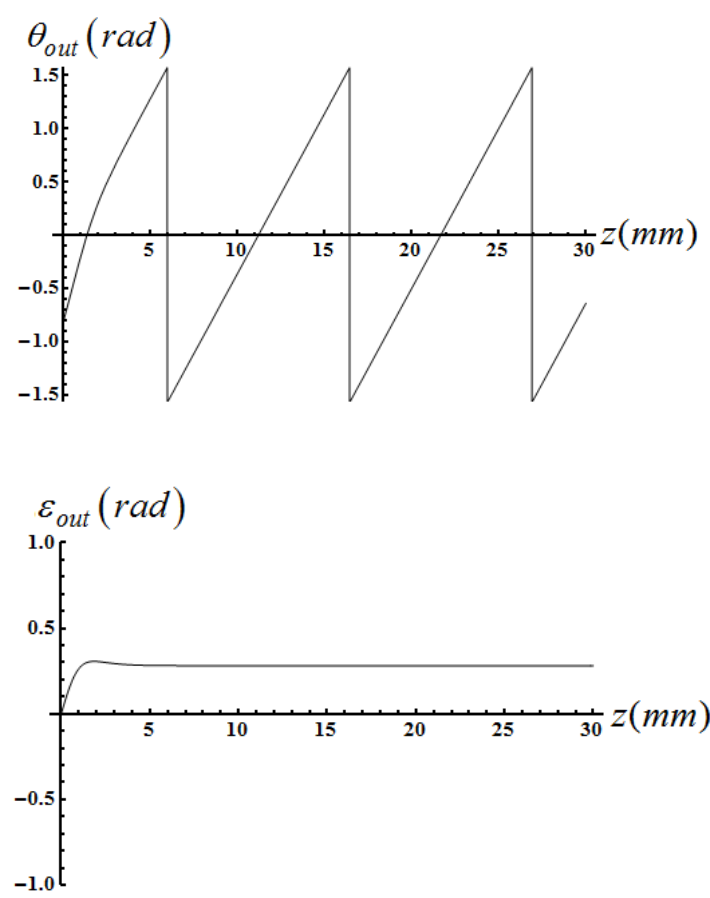

$b$

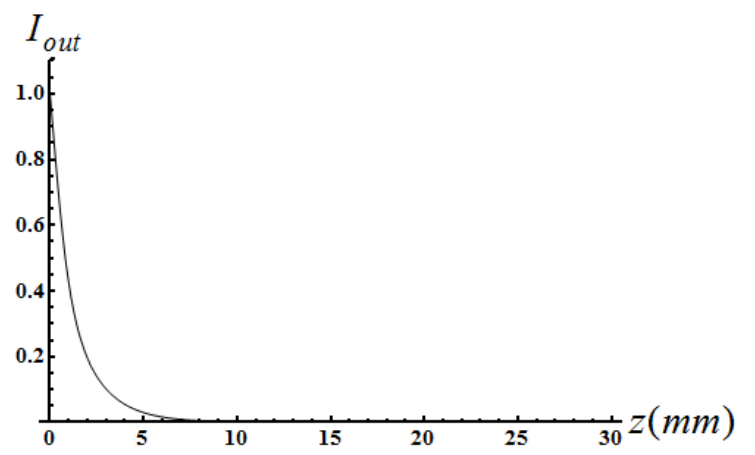

Fig. 4. Evolution of linear polarization with the azimuth $\theta_{\text {inp }}=$ $-0.8(\mathrm{rad} / \mathrm{mm})$ in longitudinally inhomogeneous medium possessing elliptical amplitude anisotropy with parameters $\xi_{0}=$ $1.22(1 / \mathrm{mm}), \theta_{0}=0.3(\mathrm{rad} / \mathrm{mm}), r_{0}=0.7(1 / \mathrm{mm})$, (a) evolution of the azimuth; (b) evolution of the ellipticity, and (c) evolution of the intensity.

Fig. 3 shows that in longitudinally inhomogeneous medium with linear dichroism depending on the ratio between the anisotropy parameters $\xi_{0}$ and $\theta_{0}$, the azimuth of input linear polarization can be changed linearly or nonlinearly. Here, the ellipticity remains unchanged (equal to zero). Fig. 4 shows that, in the medium with elliptical dichroism, the azimuth changes linearly, i.e., there is rotation of the polarization plane, and ellipticity tends effectively to some constant nonzero value depending on anisotropy of the medium. The intensity of light in both cases of media (with linear and elliptical dichroism) decreases rapidly with the coordinate $z$.

\section{Conclusions}

We show that privileged polarizations exist for both types of longitudinally inhomogeneous media: with linear and elliptical dichroism. However, unlike the case of inhomogeneous birefringence for the media with longitudinally inhomogeneous linear and elliptical dichroism, privileged polarizations are always nonorthogonal, and their azimuths do not coincide with orientation of the input molecular plane. Also, taking definite ratio between anisotropy parameters, in the case of linear dichroism the privileged polarizations can change from linear to elliptical and even become the same.

Both classes of longitudinally inhomogeneous dichroic media demonstrate orthogonalization properties. However, unlike the case of inhomogeneous birefringent medium characterized by continuum of orthogonalized polarizations, longitudinally inhomogeneous dichroic media have a finite number of orthogonalized polarizations.

Note that, when arbitrary linear polarization propagates in inhomogeneous medium with linear dichroism, it remains linear, and the azimuth changes can be both linear and non-linear. Whereas, in the case of inhomogeneous media with elliptical dichroism, the azimuth changes linearly and ellipticity tends effectively to some constant non-zero value depending on the anisotropy of the medium. In both cases, the intensity decreases rapidly with the coordinate $z$.

\section{References}

1. S. Chandrasekhar, Liquid Crystals. Cambridge University Press, Cambridge, 1977.

2. L.M. Blinov, Electro- and Magnetooptics of Liquid Crystals. Nauka, Moscow, 1978.

3. S.A. Pikin, L.M. Blinov, Liquid Crystals. Nauka, Moscow, 1982.

4. T.Z. Kosc, K.L. Marshall, A. Trajkovska-Petkoska et al. Progress in the development of polymer cholesteric liquid crystal flakes for display applications // Displays, 25 No. 5, p. 171-176 (2004).

5. B. Das, S. Vyas, J. Joseph et al., Transmission type twisted nematic liquid crystal display for three gray-level phase-modulated holographic data storage systems // Opt. and Las. Eng. 47, N. 11, p. 1150-1159 (2009).

6. P. García-Martínez, M. del Mar Sánchez-López et al., Accurate color predictability based on a spectral retardance model of a twisted-nematic liquid-crystal display // Opt. Communs. 284, Iss. 10-11, p. 2441-2447 (2011).

7. A.S. Marathay, Matrix-operator description of propagation of polarized light through cholesteric liquid crystals // J. Opt. Soc. Am. 61, p. 1363-1372 (1971). 
8. R.C. Jones, A new calculus for the treatment of optical systems. I. Description and discussion of the calculus // J. Opt. Soc. Am. 31, p. 488-493 (1941).

9. F. Perrin, Polarization of light scattering by isotropic opalescent media // J. Chem. Phys. 10, p. 415-427 (1942).

10. R.C. Jones, A new calculus for the treatment of optical systems. VII. Properties of the N-matrices // J. Opt. Soc. Am. 38, p. 671-685 (1948).

11. R.M.A. Azzam, Propagation of partially polarized light through anisotropic media with or without depolarization. A differential $4 \times 4$ matrix calculus // J. Opt. Soc. Am. 68, p. 1756-1767 (1979).

12. H. Hurwitz, R.C. Jones, A new calculus for the treatment of optical systems. II. Proof of the three general equivalence theorems // J. Opt. Soc. Am. 31, p. 493-499 (1941).

13. R.M.A. Azzam, N.M. Bashara, Simplified approach to the propagation of polarized light in anisotropic media-application to liquid crystals // $\mathrm{J}$. Opt. Soc. Am. 62, p. 1252-1257 (1972).

14. R.M.A. Azzam, N.M. Bashara, B.E. Merrill, Trajectories describing the evolution of polarized light in homogeneous anisotropic media and liquid crystals // J. Appl. Opt. 12, p. 764-771 (1973).

15. X. Zhu, Q. Hong, Y. Huang, S.-T. Wu, Eigenmodes of a reflective twisted-nematic liquid-crystal cell // J. Appl. Phys. 94, No. 5, p. 2868-2973 (2003).
16. M. Yamauch, Jones-matrix models for twistednematic liquid-crystal devices // J. Appl. Opt. 44, No. 21, p. 4484-4493 (2005).

17. I.S. Kolomiets, S.N. Savenkov, Ye.A. Oberemok, A.S. Klimov, Studying anisotropic properties of longitudinal inhomogeneous nondepolarizing media with elliptical phase anisotropy // Semiconductor Physics, Quantum Electronics \& Optoelectronics, 16, N. 4, p. 366-373 (2013).

18. M.S. Koev, I.S. Kolomiets, S.N. Savenkov, Ye.A. Oberemok, A.S. Klimov, Propagation of privileged waves in longitudinally inhomogeneous medium with linear birefringence and dichroism // Semiconductor Physics, Quantum Electronics \& Optoelectronics, 17, N. 4, p. 403-407 (2014).

19. R.M.A. Azzam, N.M. Bashara, Elipsometry and Polarized Light. New-York, 1977.

20. I.S. Kolomiets, Ye.A. Oberemok, S.N. Savenkov, Condition of orthogonality for self-polarizations for the first and second Jones equivalency theorems in the approximation of homogeneous and layered media // Metallofizika noveishiye tekhnologii, 33, special issue, p. 493-502 (2011).

21. R.M.A. Azzam, Polarization orthogonalization properties of optical systems // Appl. Phys. A, 13, p. 281-285 (1977).

22. S.N. Savenkov, Y.V. Aulin, Orthogonal properties of homogeneous anisotropy medium // Proc. SPIE, 6536, p. 65360D (2007). 